Results Social and cultural engagement were both independently associated with a lower risk of developing dementia in older age in fully-adjusted models. Using time-to-event analyses, socialising once a week was associated with a $29 \%$ lower risk $\mathrm{OR}=0.71 \quad 95 \% \mathrm{CI} \quad 0.52-0.97$ and cultural engagement every few months or more was associated with a $42 \%$ lower risk $\mathrm{OR}=0.5895 \% \mathrm{CI}$ 0.41-0.80. Using competing risk models, socialising $\mathrm{OR}=0.80 \quad 95 \% \mathrm{CI} \quad 0.59-1.08$ and cultural engagement $\mathrm{OR}=0.65$ 95\%CI $0.47-0.90$. Using modified Fine and Gray Subdistribution hazards models, socialising $\mathrm{OR}=0.66$ 95\%CI $0.53-0.82$ and cultural engagement $\mathrm{OR}=0.4295 \% \mathrm{CI}$ $0.32-0.56$. Community group activities were only associated with dementia in minimally-adjusted models. Results were robust to sensitivity analyses considering moderators, reverse causality, over-adjustment, and baseline cognitive function.

Conclusion The results presented here suggest that social and cultural engagement are independent risk-reducing factor for the development of dementia in older age. Even for those who lack contact with friends and family or who socialise infrequently, engagement with cultural venues, even on a less frequent basis, could be protective against the incidence of dementia. These findings align with broader findings relating to cognitive reserve and support the development of multimodal community-based interventions to promote healthy cognitive ageing amongst older adults.

\section{OP25 THE ASSOCIATION OF POOR ORAL HEALTH WITH A RANGE OF INFLAMMATORY MARKERS: RESULTS FROM TWO POPULATION BASED STUDIES OF OLDER PEOPLE IN THE UK AND USA}

\begin{abstract}
${ }^{1} \mathrm{E}$ Kotronia*, ${ }^{2} \mathrm{GS}$ Wannamethee, ${ }^{2} \mathrm{OA}$ Papacosta, ${ }^{3} \mathrm{PH}$ Whincup, ${ }^{2} \mathrm{LT}$ Lennon, ${ }^{4} \mathrm{RJ}$ Weyant, ${ }^{5}$ TB Harris, ${ }^{1,2}$ SE Ramsay. 'Institute of Health and Society, Newcastle University, Newcastle Upon Tyne, UK; ${ }^{2}$ Department of Primary Care and Population Health, UCL, London, UK; ${ }^{3}$ Population Health Research Institute, St George's University of London, London, UK; ${ }^{4}$ Department of Dental Public Health, School of Dental Medicine, University of Pittsburgh, Pittsburgh, USA; ${ }^{5}$ Laboratory of Epidemiology and Population Sciences, National Institute of Aging, Bethesda, USA
\end{abstract}

\subsection{6/jech-2019-SSMabstracts.25}

Background Oral health conditions such as tooth loss, periodontal (gum) disease and dryness of mouth are very common health problems in older people, with significant impacts on nutrition, quality of life and well-being of ageing populations. Oral health, particularly periodontal disease, has been linked to systemic inflammation, such as high C-reactive protein (CRP) and interleukin-6 (IL-6). However, very few studies of older people have investigated the associations between oral health and inflammation. We examined whether a range of oral health markers are associated with inflammatory markers in two population-based studies of older people in the UK and USA.

Methods Cross-sectional analyses were conducted in the British Regional Heart Study (BRHS) comprising men aged 71-92 $(n=2147)$ from 24 British towns, and the US Health, Aging and Body Composition (HABC) Study comprising men and women aged 71-80 $(n=3075)$. Assessments included oral health (periodontal disease, tooth count, dry mouth) and inflammatory markers such as CRP, IL-6, leptin, tissue plasminogen activator (tPA), von Willebrand Factor (vWF), fibrin Ddimer, high sensitivity Troponin $\mathrm{T}$ (hsTnT), N-Terminal prohormone of brain natriuretic peptide (NTproBNP), and proinsulin.
Results In the BRHS, having no natural teeth was associated with being in the top tertiles of CRP, and fibrin D-dimer (odds ratio $(\mathrm{OR})=1.35,95 \% \mathrm{CI}$ : $1.01-1.80 ; \mathrm{OR}=1.38,95 \%$ CI: 1.03-1.85, respectively) after adjustment for age, social class, smoking, history of cardiovascular disease and diabetes, and BMI. Tooth loss ( $<21$ teeth) was associated with being in the top tertiles of CRP, hsTnT, fibrin D-dimer, and NTproBNP (fully adjusted $\mathrm{OR}=1.31$, 95\%CI: 1.02-1.68; $\mathrm{OR}=1.32$, 95\%CI: $1.01-1.74 ; \mathrm{OR}=1.37$, 95\%CI: $1.05-1.77$, $\mathrm{OR}=1.40$, 95\% CI: 1.01-1.94, respectively). Periodontal disease was associated with being in the top tertile of hsTnT. In the HABC Study, having no teeth and partial tooth loss were associated with being in the top tertile of CRP $(\mathrm{OR}=1.57$, 95\% CI: 1.10-2.25; $\mathrm{OR}=1.40,95 \% \mathrm{CI}:$ 1.13-1.75, respectively) after adjustment for age, gender, race, education, smoking, history of cardiovascular disease and diabetes, and BMI. Moreover, having $\geq 3$ oral health problems was associated with being in the top tertiles of CRP and IL-6 after full adjustment.

Conclusion Poor oral health in older people was associated with increased levels of various inflammatory markers including CRP, fibrin D-dimer, hsTnT and NTproBNP. These findings suggest that poor oral health in older age is linked not only to general systemic inflammation but also inflammation associated with metabolic disturbances. These associations could offer insights into mechanistic pathways by which poor oral health could influence age-related conditions.

\section{Mental Health 1}

\section{OP26 PATIENT EXPERIENCE OF ENGAGEMENT WITH HEALTHCARE SERVICES FOLLOWING AN EPISODE OF HIGH RISK SELF-HARM: A MIXED METHODS STUDY}

\footnotetext{
${ }^{1,2} \mathrm{G}$ Cully, ${ }^{1,2} \mathrm{D}$ Leahy, ${ }^{1,3} \mathrm{~F}$ Shiely, ${ }^{1,2} \mathrm{E}$ Arensman*. 'School of Public Health, University College Cork, Cork, Ireland; ${ }^{2}$ National Suicide Research Foundation, Cork, Ireland; ${ }^{3}$ HRB Clinical Research Facility, Mercy University Hospital, Cork, Ireland
}

\subsection{6/jech-2019-SSMabstracts.26}

Background Hospital management of self-harm is an essential component of suicide prevention. Patients presenting to hospital for self-harm involving highly lethal methods or with high suicidal intent are a subgroup of self-harm patients at high risk of suicide. Investigating healthcare service provision from the patients' perspective is integral to the design and implementation of better quality care. The current study explored patients' experiences of engaging with healthcare services after a high risk self-harm presentation to hospital.

Methods A sequential transformative mixed-methods design was used. Quantitative information was obtained by interview administered questionnaires (including internationally validated scales, closed and open-ended questions) 0-3 months following a high risk self-harm presentation to a hospital emergency department $(n=67)$. Semi-structured follow-up interviews were conducted 6-9 months later providing qualitative data $(n=31)$. Follow-up interviews were recorded, transcribed and thematically analysed using NVivo software. Quantitative information was analysed in SPSS Version 25. Both methodologies were integrated during the interpretation of the results.

Results After the self-harm presentation, 85.7\% of patients reported receiving follow-up care with public outpatient mental health services and $61.8 \%$ attended their general 